\title{
The influence of process parameters on the laser-induced coloring of titanium
}

\author{
Arkadiusz J. Antończak • Bogusz Stępak • \\ Paweł E. Kozioł • Krzysztof M. Abramski
}

Received: 18 March 2013 / Accepted: 12 August 2013 / Published online: 28 August 2013

(c) The Author(s) 2013. This article is published with open access at Springerlink.com

\begin{abstract}
This paper presents the results of the measurements and analysis of the influence of laser process parameters on the color obtained. The study was conducted for titanium (Grade 2) using a commercially available industrial pulsed fiber laser. It was determined how a variety of different laser process parameters, such as laser power, the scanning speed of the material, the temperature of the material, the size of the marked area, and the position of the sample, relative to both the focal plane and the center of the working field of the system, affect the repeatability of the colors created. For an objective assessment of color changes, an optical spectrometer and the CIE color difference parameter $\Delta E_{a b}^{*}$ were used. Our paper explains why the tolerance of process parameters highly depends on the specific color. Additionally, a comparison of the results for titanium with those obtained for stainless steel in a previous study is presented. Based on this analysis, a number of necessary modifications are proposed to laser systems commonly used for monochrome marking in order to improve repeatability in color marking.
\end{abstract}

\section{Introduction}

Titanium oxide coatings have a variety of useful physical properties. Applications include electrodes for photovoltaic cells [1], environmental purification, sterilization, and cancer therapy (for which the material is an excellent pho-

A.J. Antończak $(\bowtie) \cdot$ B. Stępak · P.E. Kozioł · K.M. Abramski Laser and Fiber Electronics Group, Department of Electronics, Wroclaw University of Technology, Wyb. Wyspianskiego 27,

50-370 Wroclaw, Poland

e-mail: arkadiusz.antonczak @ pwr.wroc.pl

Fax: +48-713-203189 tocatalyst [2]), gas sensing, in which chemisorption nanocrystalline thin films are used [3], bone implants (biocompatible material) [4], and optical components for which protective and antireflective coatings are required [5]. Another interesting feature of such coatings is related to their visual aspect. Titanium oxide films, present a range of colors, which allows them to be used in decorative applications [6], electronics, the automotive industry, and art-for new designs, advertising, and jewelry $[7,8]$.

Various techniques can be used to obtain a titanium oxide coating: cathodic deposition [9], plasma deposition [10], laser deposition [11], magnetron sputtering [12], thermal oxidation [13], electrolytic oxidation (anodizing) [7, 14], and laser oxidation $[8,14,15]$.

Most frequently, color markings on metal surfaces are made by printing, emulsion coating, or electrolytic oxidation techniques. Poor scratch properties and wear resistance, the complexity of the process and color fading over time are typical drawbacks of printing methods. Anodizing, in turn, does not allow either selective marking or more than one color at a time. Laser color marking as a process has been known for over 15 years [16], but it has not been used widely in the industry as it is regarded as difficult, time-consuming, and most importantly, not repeatable. The two main methods of laser coloring of metals have been approached in published literature. The first utilizes a laser as a heat source, which allows a transparent or semitransparent oxide film to form on the metal surface $[6,8,14,17,18]$. White light illuminating the sample is reflected from the surface of both the oxide and metal. As a result of interference of the reflected beams, a color effect can be obtained. The thickness of the oxide layer, the order of interference [19], and its refractive index determine the color spectrum. Secondly, color can be obtained on the surface of various metals by the laser-induced formation of periodic surface structures (LIPSS), known as 
"laser-induced ripples," on the surface created using femto or picosecond lasers [20-23]. Interestingly, the structure of this type may also be prepared in liquids [23].

In most applications, laser marking is the fastest and the cheapest method. There is a strong demand to extend the capabilities of these systems with respect to the possibility of color marking. The two main problems are the reproducibility and stability of the process. Often, experimentally determined parameters required to obtain a specific color on one system, do not give a positive result for other systems, even very similar (or the same model). Several descriptions of laser color formation on a metal surface can be found in literature, but there is still a lack of information about which of the process parameters have an impact on the reproducibility of the colors created, and to what extent.

\section{Experiment details}

The study was performed for the commonly used MOPA configuration Yb:glass fiber laser $(1062 \mathrm{~nm})$ with average output power $20 \mathrm{~W}$, beam quality factor $M^{2} \leq 1.5$, pulse duration $230 \mathrm{~ns}$, and pulse repetition rate $20-80 \mathrm{kHz}$ (IPG type YLP-C-1-100-20-20).

The system was equipped with galvanometric scan mirrors that allow the beam to be deflected. The laser beam was focused on the target through a $160 \mathrm{~mm}$ focal length F-Theta lens (LINOS type 4401-305-000-21). The beam diameter at the focal point was approximately $40 \mu \mathrm{m}$. Samples of the materials used for marking were placed on an adjustable ( $Z$-axis) table. A block diagram of the diagnostic system is shown in Fig. 1.

An external distance sensor allowed the displacement of the sample in the $Z$-axis to be measured with an accuracy of $10 \mu \mathrm{m}$. A thermo-electric cooler (TEC) and a temperature controller (ILX Lightwave type LDT-5525) were used for changing the temperature of the marked plates with a resolution of $0.1^{\circ} \mathrm{C}$.

Tests were carried out on plates made of the most commonly used type of titanium, the grade 2 (chemical composition: $\mathrm{Ti}=99.2 \%, \mathrm{Fe}=0.3 \%_{\max }, \mathrm{O}=0.25 \%_{\max }$, $\left.\mathrm{C}=0.08 \%_{\max }, \mathrm{N}=0.03 \%_{\max }, \mathrm{H}=0.02 \%_{\max }\right)$ with dimensions of $100 \times 100 \mathrm{~mm}$ and thickness of $1 \mathrm{~mm}$. Before the experiment, the plates were washed with isopropyl alcohol in an ultrasonic cleaner. The samples were marked in atmospheric air.

For an objective evaluation of the color changes due to the changes in process parameters, an optical spectrometer (Ocean Optics type USB 4000) was used. The light source was a GrafiLite lamp of True Color type (color rendering in$\operatorname{dex} C R I=82$, color temperature $T_{C}=5600 \mathrm{~K}$ ). This lamp was also used when taking the pictures of samples.

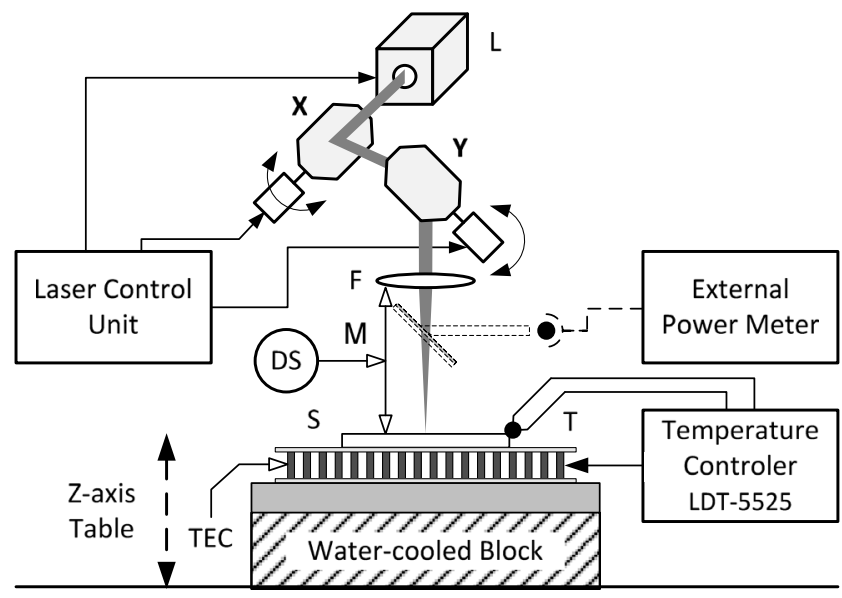

Fig. 1 Block diagram of the system used for laser-induced coloring, where: L-laser; F-F-Theta lens; DS — distance sensor; T-temperature sensor; TEC-thermo-electric cooler; $\mathrm{S}$-tested sample; $X, Y$, $Z$-axes of the system

The software used to control the spectrometer (Spectra Suite) enables operation on several color spaces. For colorimetric identification, one of the most useful color spaces, the CIE $L^{*} a^{*} b^{*}$, was used. It enables color difference to be easily evaluated. The CIE $L^{*} a^{*} b^{*}$ color space is organized in a cube form and is almost uniform. Its unit vector is approximately identical to the color resolution of the human eye.

As the perceived color partly depends on the angle of observation [19], many measurements were performed in order to find the optimal angle. The similarity of the color perceived on the metal plate and the one generated on the basis of the measured $L^{*} a^{*} b^{*}$ data was adopted as a criterion for the optimal angle. On this basis, an angle of about $\alpha=60{ }^{\circ} \mathrm{C}$ was chosen, and this was kept constant for all further measurements. The measuring fiber was completed with a collimating lens (Ocean Optics type 74-VIS; 0.35$2 \mu \mathrm{m})$. The collimator's distance to the surface of the plate was fixed at $10 \mathrm{~mm}$ (the focal length of the collimator). In the CIE $L^{*} a^{*} b^{*}$ color space, the difference between the two measured colors can be expressed by the CIE color difference formula [24]:

$\Delta E_{a b}^{*}=\sqrt{\left(\Delta L^{*}\right)^{2}+\left(\Delta a^{*}\right)^{2}+\left(\Delta b^{*}\right)^{2}}$

where $\Delta E_{a b}^{*}$ (total color difference) is the Euclidean distance between two points in a three-dimensional color space, and $\Delta L^{*}, \Delta a^{*}$, and $\Delta b^{*}$ indicate how much a standard and a sample differ from one another in $L^{*}, a^{*}$, and $b^{*}$, respectively. The theory is that a value of $\Delta E_{a b}^{*}=1$ corresponds to the smallest color difference that a statistical human eye can recognize. In practice, the $\Delta E_{a b}^{*}$ values of 4 and over are normally recognized by an average person, while a difference of $\Delta E_{a b}^{*}=2$ is perceived only by an experienced observer. Statistical studies indicate that a color difference 
$\Delta E_{a b}^{*}$ of 6 to 7 is often considered as "acceptable" to buyers of print materials [25]. After analyzing the results of laser color marking, it was decided to apply the same scale to assess the similarity of the color obtained. The same criterion was also used in previous work [18]. This approach allowed both cases to be compared against each other. To determine the reproducibility and the measurement error of $\Delta E_{a b}^{*}$, statistical measurements of selected samples were performed. By measuring the same sample 25 times, the average value of the $\Delta E_{a b}^{*}$ parameter was determined as 1.1. The variance of the measurement error value was 0.11. Assuming that the established limit of the acceptability of color difference is $\Delta E_{a b}^{*}=7$, we concluded that the aforementioned measurement method for color recognition is reproducible.

\section{Laser-induced color marking parameters}

In order to find obtainable colors, the study began with a matrix test. Matrix methods allowed simultaneous analysis of the impact of two process parameters, e.g., the laser beam power and the scanning speed of the sample. The influence of both parameters on the color palette for the two different hatching values $h=5$ and $10 \mu \mathrm{m}$ (defined as a distance between adjacent traces) is shown in Fig. $2 a$ and $2 b$.

The tested titanium plates contained up to 64 fields with dimensions of $5 \times 5 \mathrm{~mm}$ and about $2 \mathrm{~mm}$ interval between successive rows and columns. Each tested field corresponded to a different set of process parameters (laser fluence). The tests were conducted for the experimentally determined range of parameters (presented in Table 1).

The colors obtained for a smaller raster distance were more saturated. However, due to the extremely long times required by the process at low speeds, the hatching value of $10 \mu \mathrm{m}$ was chosen for further testing. The initial test allowed us to find a dozen colors, of which the six most representative are shown in Fig. 2c. The parameters corresponding to these colors (after small adjustments) are shown in Table 2. The value of the accumulated fluence (during the scanning of one line) was determined as

$F=\frac{2 \sqrt{2} P}{\pi \omega_{0} V}$

where $P$ is the average laser power, $\omega_{0}$ is the radius of the laser beam at the focal point, and $V$ is the scanning speed of the sample.

With increasing laser fluences, the temperature in the irradiated area of sample rises, which enhances the diffusion of oxygen into the surface and the oxidation reaction. However, for large accumulated fluences, oxide growth can be affected by evaporation and particle ejection resulting in decreased coating thickness [26]. In the case under analysis, the use of a pulsed fiber laser and exceeding the accumulated

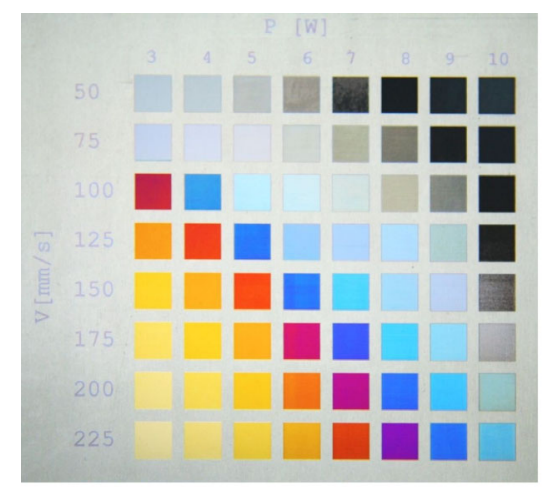

$\mathbf{a}$

$\mathbf{a}$

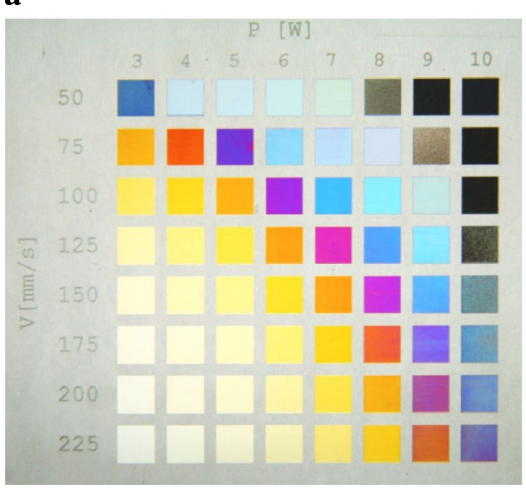

b

Fig. 2 The influence of average power and scanning speed of the sample on the color: (a) example for pulse repetition rate $P R R=80 \mathrm{kHz}$ and hatching $h=5 \mu \mathrm{m}$, (b) example for $P R R=80 \mathrm{kHz}$ and $h=10 \mu \mathrm{m}$, (c) colors selected for the study

Table 1 Parameters selected for testing of laser color marking

\begin{tabular}{llll}
\hline Average laser power & $P[\mathrm{~W}]$ & $3-10$ in steps of 1 \\
Speed of scanning & $V[\mathrm{~mm} / \mathrm{s}]$ & $50-225$ in steps of 25 \\
Pulse repetition rate & $P R R[\mathrm{kHz}]$ & 80 & 10 \\
Hatching & $h[\mu \mathrm{m}]$ & 5 & \\
\hline
\end{tabular}

fluence of about $420 \mathrm{~J} / \mathrm{cm}^{2}$ caused ablation of the material, which is shown in Fig. 3e. Due to the different mechanism of formation, the colors obtained above the ablation threshold, for example, silver and black (see Fig. 2a and b, and Table 2), have been omitted and excluded from the colorimetric analysis.

In order to check the possibility of obtaining other colors (e.g., green) and to avoid the ablation threshold, a test consisting of $\mathrm{N}$-fold irradiation of the same area with a constant, low value of laser fluence $\left(45 \mathrm{~J} / \mathrm{cm}^{2}\right)$ was performed. The results are shown in Fig. 3f.

This method enabled the same colors to be obtained, and interestingly, in the same color sequence. However, the passivation layer of titania made in this way (visible in the fields from 16 to 25 in Fig. 3f) prevented further oxidation of the titanium and made it impossible to achieve thicker layers of the oxide (other colors). As can be seen in the pictures 
Table 2 Process parameters corresponding to the selected colors and their measured color space coordinates

\begin{tabular}{|c|c|c|c|c|c|c|c|c|c|c|}
\hline \multirow[t]{2}{*}{ No. } & \multirow[t]{2}{*}{ Color } & \multirow[t]{2}{*}{ Power [W] } & \multirow[t]{2}{*}{ Speed $[\mathrm{mm} / \mathrm{s}]$} & \multirow[t]{2}{*}{$P R R[\mathrm{kHz}]$} & \multirow[t]{2}{*}{ Hatching $[\mu \mathrm{m}]$} & \multirow[t]{2}{*}{ Fluence $\left[\mathrm{J} / \mathrm{cm}^{2}\right]$} & \multicolumn{3}{|c|}{ CIE coordinates } & \multirow{2}{*}{$\begin{array}{l}\text { Marking time } \\
\text { [min: } \mathrm{s}]\end{array}$} \\
\hline & & & & & & & $L^{*}$ & $a^{*}$ & $b^{*}$ & \\
\hline 1 & Yellow & 7 & 200 & 80 & 10 & 157.6 & 78.6 & 8.3 & 62.5 & $3: 20$ \\
\hline 2 & Orange & 7 & 145 & & & 216.1 & 43.1 & 40 & 60.7 & $4: 34$ \\
\hline 3 & Red & 7 & 125 & & & 252.1 & 28.4 & 55.5 & 2.9 & $5: 08$ \\
\hline 4 & Purple & 7 & 120 & & & 262.6 & 15.2 & 60.3 & -56.1 & $5: 33$ \\
\hline 5 & Blue & 7 & 100 & & & 315.1 & 29.6 & 3.6 & -73.3 & $6: 40$ \\
\hline 6 & L. Blue & 8 & 100 & & & 360.1 & 69.3 & -17.7 & -30.9 & $6: 40$ \\
\hline 7 & Silver & 9 & 75 & & & 540.2 & - & - & - & $8: 53$ \\
\hline 8 & Black & 9 & 25 & & & 1620.6 & - & - & - & $26: 40$ \\
\hline 9 & $\operatorname{Red}^{\mathrm{b}}$ & 43.8 & 781.3 & 500 & & 252.1 & - & - & - & $0: 49$ \\
\hline
\end{tabular}

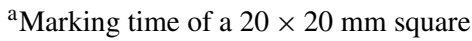

${ }^{\mathrm{b}}$ The case was considered theoretically — see text (the estimation does not include amendments of the accumulative thermal effect due to the changes of the scanning speed of the sample)
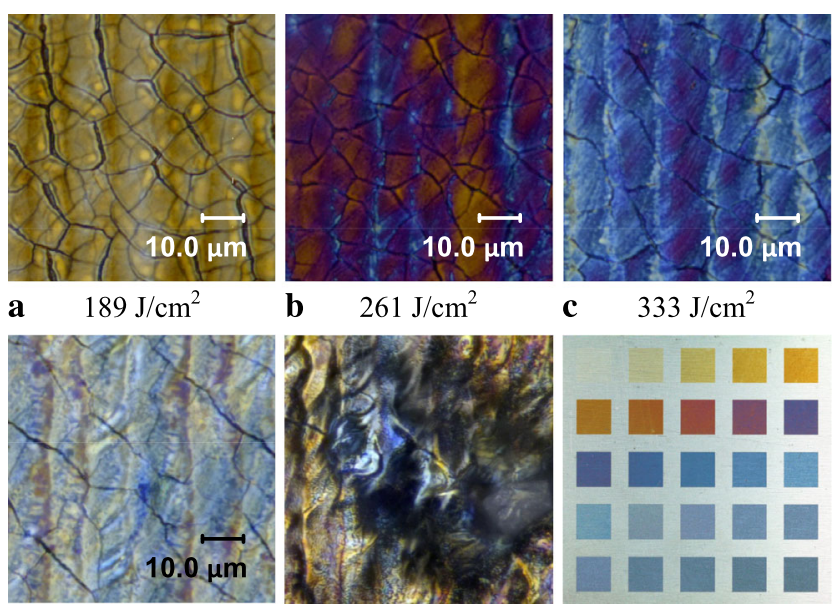

d $405 \mathrm{~J} / \mathrm{cm}^{2}$

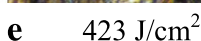

f $\mathrm{N} \times 45 \mathrm{~J} / \mathrm{cm}^{2}$

Fig. 3 The microscope images of titanium oxide film produced on titanium substrate for different value of the accumulated laser fluence: (a) surface morphology of the sample irradiated at: $189 \mathrm{~J} / \mathrm{cm}^{2}$, (b) $261 \mathrm{~J} / \mathrm{cm}^{2}$, (c) $333 \mathrm{~J} / \mathrm{cm}^{2}$, (d) $405 \mathrm{~J} / \mathrm{cm}^{2}$, (e) $423 \mathrm{~J} / \mathrm{cm}^{2}$ (above the ablation threshold), (f) sample with $\mathrm{N}$-fold repeated constant value $\left(45 \mathrm{~J} / \mathrm{cm}^{2}\right)$ of the laser fluence (the total dose of fluence at each field of the sample is equal to the product of the positions of the marked area and the laser fluence $\left(45 \mathrm{~J} / \mathrm{cm}^{2}\right)$ - order from left to right and from top to bottom). Images $4 \mathrm{a}-\mathrm{e}$ were taken with a digital microscope; the Keyence VHX-1000E. Color of the images: 4a-e differs from the actual, because of the way the sample was illuminated

(Fig. 3a-d), the titania film on the titanium surface breaks during calcinations to form agglomerates with an average size of $10-20 \mathrm{~nm}$. The cracks result from the very high cooling rate of the melted pool after the laser beam moves [27]. The furrows visible in these photographs are due to the raster scanning of the material with a hatching of $10 \mu \mathrm{m}$. It should also be noted that color laser marking is relatively time consuming (Table 2). However, the use of lasers with a higher pulse repetition rate or $\mathrm{CW}$ lasers allows one to shorten the process a few times.

\section{Repeatability of laser color marking}

The first tests of laser color marking that we performed were not reproducible. We therefore decided to carry out a detailed study of which parameter determines the final result and to what extent. Measurements of the repeatability of laser color marking were made as a function of the initial temperature of the material $\left(1-40^{\circ} \mathrm{C}\right)$, the location of the marking surface relative to the focal point $( \pm 1.2 \mathrm{~mm})$, the fluctuation of average power $( \pm 0.2 \mathrm{~W}$, which corresponds to $\pm 2.9 \%$ of the reference power), the speed of scanning of the surface $( \pm 10 \mathrm{~mm} / \mathrm{s})$, the position of the marking field relative to the centre of the working field of the system $( \pm 30 \mathrm{~mm})$-we called this the "galvanometric scanning effect" - and the size of the marked field $(5 \times 5$ to $14 \times 14 \mathrm{~mm}$ ), which enables the investigation of the accumulative thermal effects.

Since the main reason (apart from oxygen) for the formation of oxides on the material surface is temperature, its impact on the obtained colors was investigated: The results are shown in Fig. 4a.

The temperature of the metal plate was controlled using a thermoelectric element TEC. Each time (during testing) a marked area was placed in the centre of the working field. The results of colorimetric measurements are shown in two forms: Fig. $4 \mathrm{~b}$ is a visual presentation (the color palette created based on the $L^{*}, a^{*}, b^{*}$ parameters) and Fig. $4 \mathrm{c}$ is a graph of the total color difference $\Delta E_{a b}^{*}$ as a function of temperature. All the colors tested were compared to the reference colors indicated in Fig. 4b. Although the resulting 

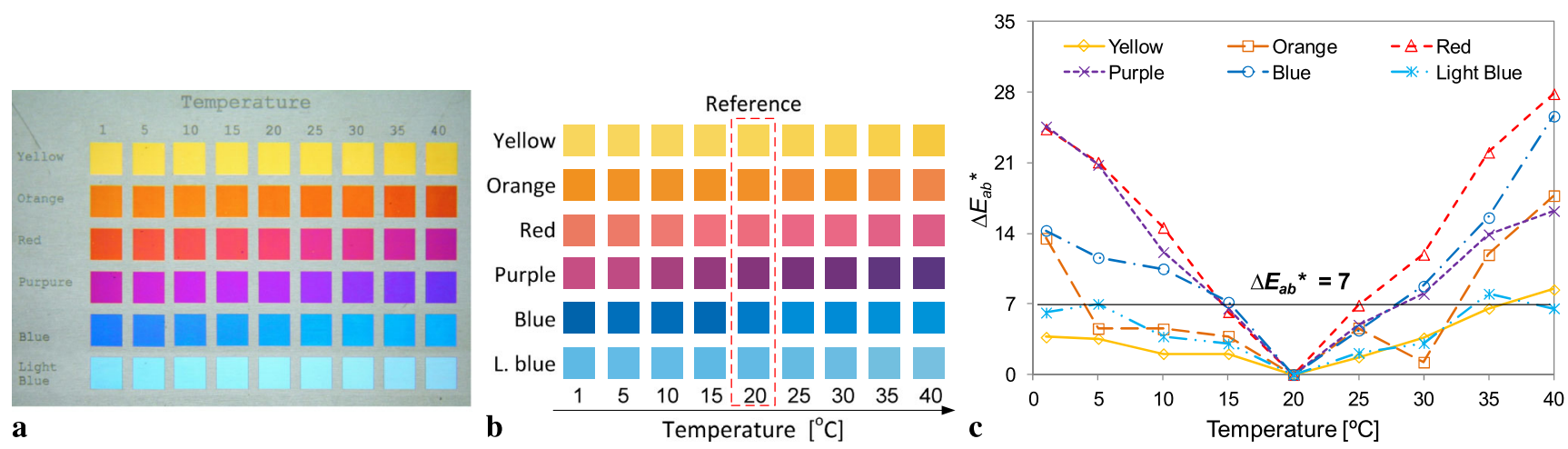

Fig. 4 The influence of the initial temperature of the material on the colors obtained: (a) photograph of the test sample, (b) the color palette created based on the colorimetric measurements, (c) graph of the $\Delta E_{a b}^{*}$ parameter

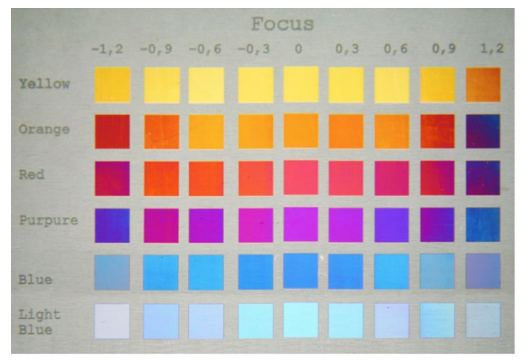

$\mathbf{a}$

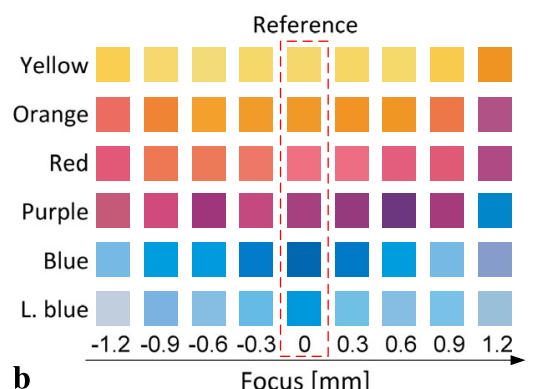

Focus $[\mathrm{mm}]$

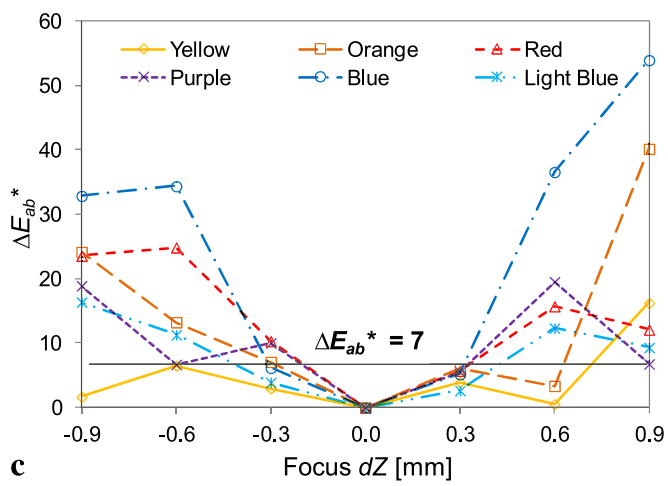

Fig. 5 The influence of material position relative to the focal point on the obtained color: (a) photo of the test sample, (b) the color palette created on the basis of colorimetric measurements, (c) graph of the $\Delta E_{a b}^{*}$ parameter

shades of each color appear to be quite similar (Fig. 4b), the colorimetric assessment clearly defines (for a fixed value of $\left.\Delta E_{a b}^{*}=7\right)$ the allowable temperature range for each color. The smallest tolerances were observed for red and purple $\left(\leq \pm 5^{\circ} \mathrm{C}\right)$. Yellow and light blue, in turn, displayed a significantly higher resistance to the changes of the initial temperature of the material $\left(\leq \pm 15^{\circ} \mathrm{C}\right)$. The explanation of this situation, as well as the influence of other process parameters on the resulting color (due to some analogies) will be discussed in the next section.

A further experiment which gained interesting results analyzed the impact of a marked surface position relative to the waist of the laser beam. The results are presented in Fig. 5 .

In this case, the color obtained was found to correlate strongly with the position of the plate. As expected, the dependence of the $\Delta E_{a b}^{*}$ error is almost symmetric with respect to the point of focus. Given the previously accepted value of the $\Delta E_{a b}^{*}$ parameter, the permissible deviation of the position of the marked surface relative to the focal plane is $\pm 0.2-0.7 \mathrm{~mm}$, depending on the color. As before, the largest acceptable deviation was for yellow, and the smallest for red and purple. Considering the dimensioning accuracy of the marked items, this range is relatively small. In prac- tice, this means that the real distance to the target needs to be measured (e.g., with a noncontact sensor).

The changes in optical laser power or scanning speed of the material are the main source of the laser fluence fluctuation on the object being processed. Therefore, the sensitivity of color changes as a function of both these parameters had to be examined. Measurements were performed for each tested color. Selected cases are shown in Figs. 6, 7 and 8.

Colorimetric analysis of the results presented in Figs. 6c$8 \mathrm{c}$ shows that the areas (islands) with a color difference $\Delta E_{a b}^{*} \leq 7$ are spread almost symmetrically with respect to the line of constant laser fluence. Some inhomogeneities in the colorimetric image may result from samples adhering unevenly to the thermally stabilized base and from minimal deformation and/or nonuniform thickness (tolerance of $50 \mu \mathrm{m}$ ) of the material. This, however, had no significant effect on the dominant, visible trend.

Similarly to the previous cases, the sensitivity to average power or scanning speed changes has a much more significant impact for the red and blue colors than for the yellow. For yellow, a fluctuation of about $0.2 \mathrm{~W}(\sim 3 \%)$ did not induce large color changes, whereas for red or blue, the maximum power change should not exceed $0.07 \mathrm{~W}(\sim 1 \%)$. 


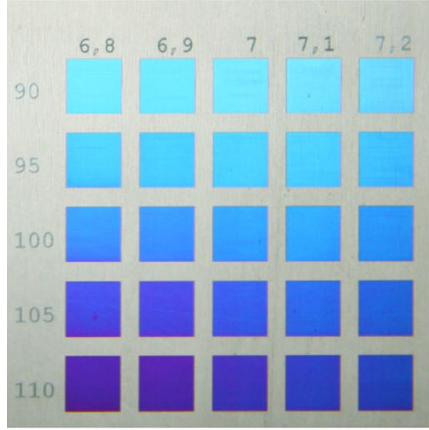

$\mathbf{a}$
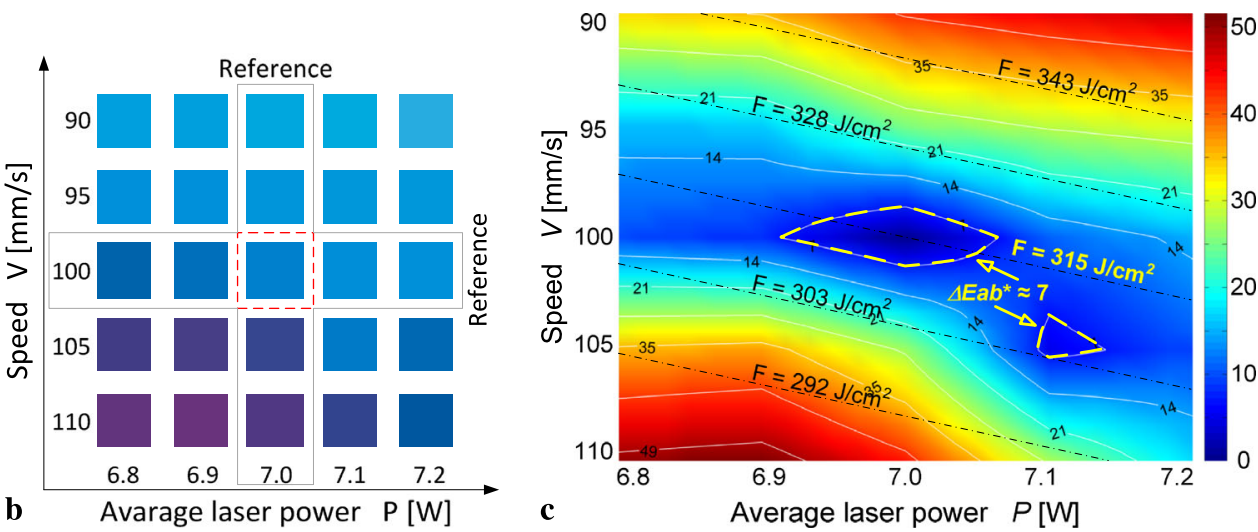

Fig. 6 The influence of the average power and speed of scanning of the sample on the blue color (the reference parameters for the blue color are $P=7 \mathrm{~W}, V=100 \mathrm{~mm} / \mathrm{s}, F_{R}=80 \mathrm{kHz}, h=10 \mu \mathrm{m}$ ):

(a) photo of the test sample, (b) the color palette created based on the colorimetric measurements, (c) diagram of the $\Delta E_{a b}^{*}$ parameter

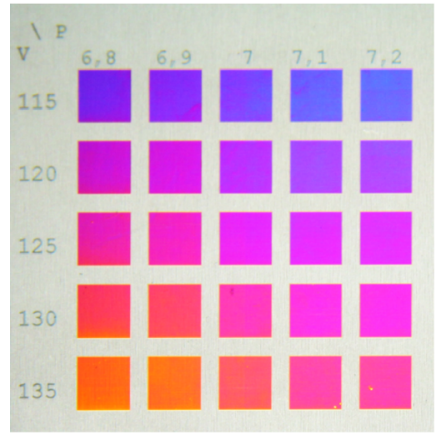

a
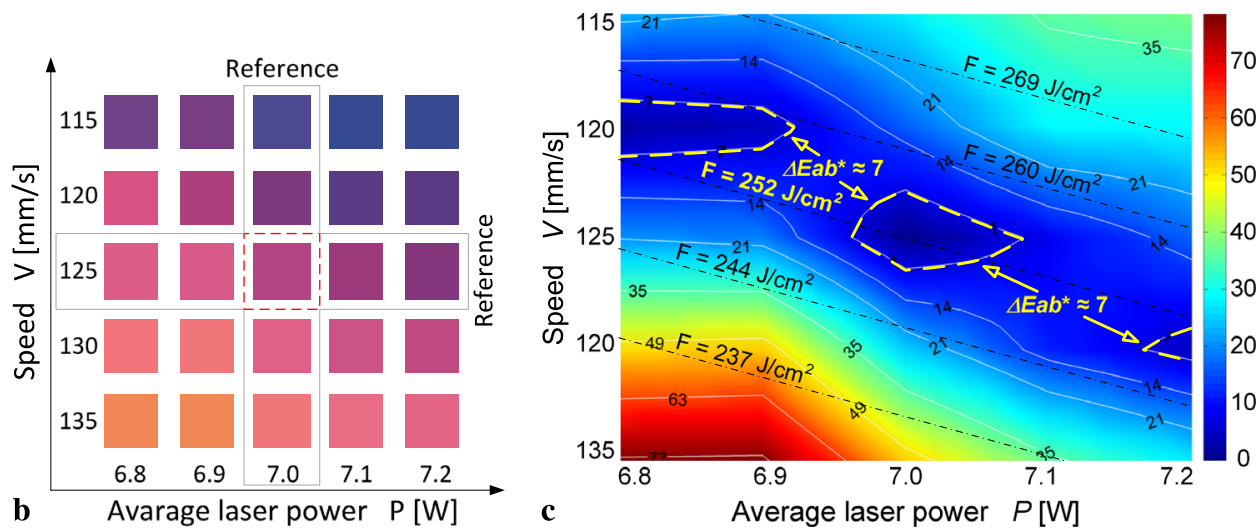

Fig. 7 The influence of the average power and speed of scanning of the sample on the red color (the reference parameters for the red color are $P=7 \mathrm{~W}, V=125 \mathrm{~mm} / \mathrm{s}, F_{R}=80 \mathrm{kHz}, h=10 \mu \mathrm{m}$ ): (a) photo of

the test sample, (b) the color palette created based on the colorimetric measurements, (c) diagram of the $\Delta E_{a b}^{*}$ parameter

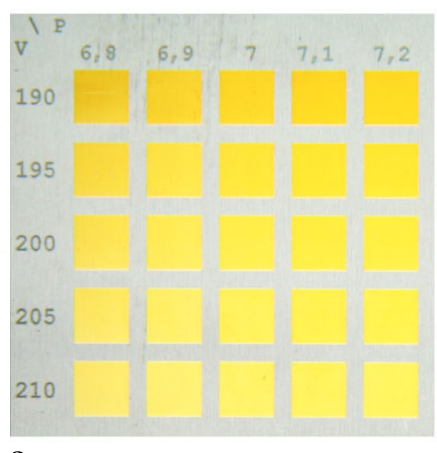

$\mathbf{a}$
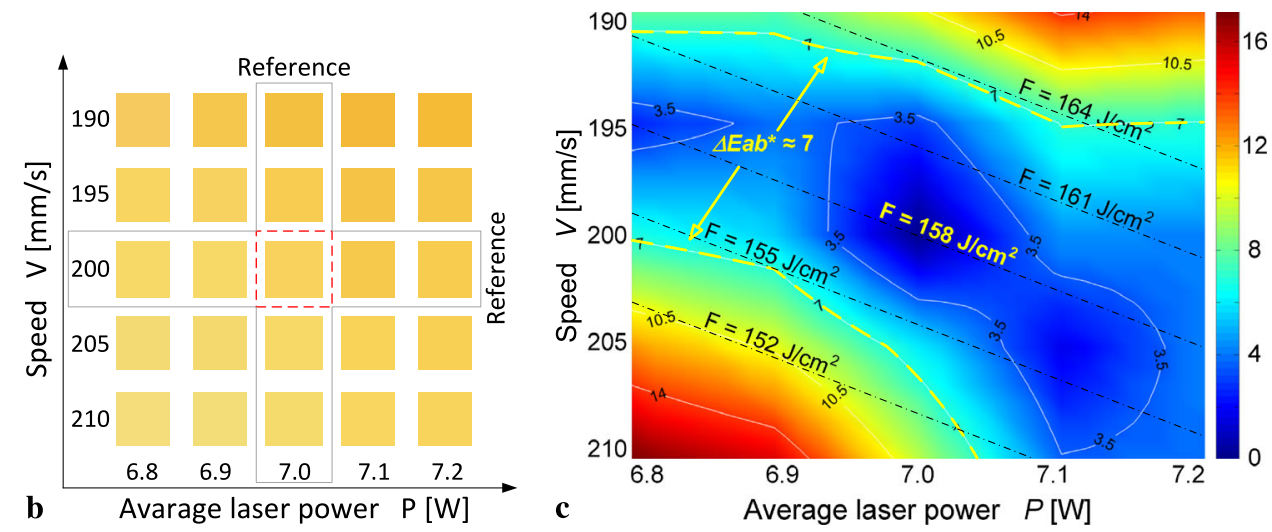

Fig. 8 The influence of the average power and speed of scanning of the sample on the yellow (gold) color (the reference parameters for the yellow color are $\left.P=7 \mathrm{~W}, V=200 \mathrm{~mm} / \mathrm{s}, F_{R}=80 \mathrm{kHz}, h=10 \mu \mathrm{m}\right)$ :

It is worth noting that the required repeatability of power exceeds the long-term stability of the optical power of this type of lasers $(<5 \%)$ [28]. This problem can be solved

(a) photo of the test sample, (b) the color palette created based on the colorimetric measurements, (c) diagram of the $\Delta E_{a b}^{*}$ parameter

by controlling the output power continuously and keeping it constant using an additional feedback system (taking into account the time-varying attenuation of the beam delivery 


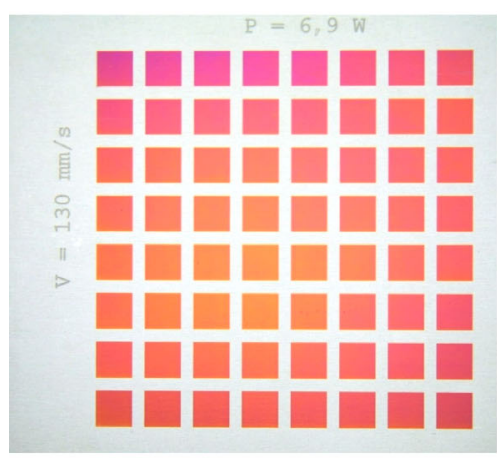

a
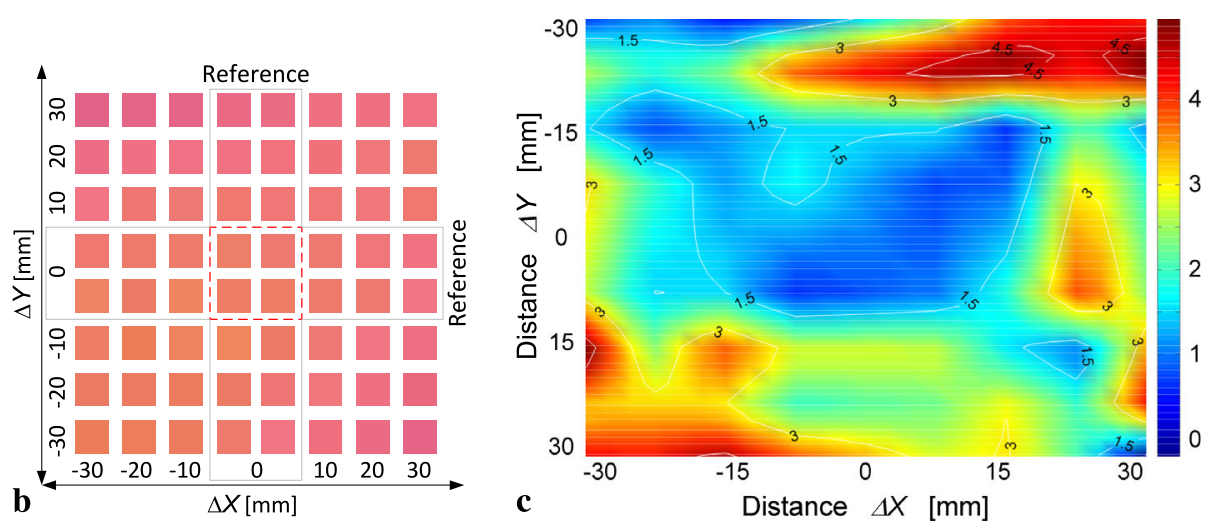

Fig. 9 The influence of position of the marked field within the working area (the reference parameters for the red color are $P=6.9 \mathrm{~W}$, $\left.V=130 \mathrm{~mm} / \mathrm{s}, F_{R}=80 \mathrm{kHz}, h=10 \mu \mathrm{m}\right)$ : (a) photo of the test sample, (b) the color palette created based on the colorimetric measurements, (c) diagram of the $\Delta E_{a b}^{*}$ parameter

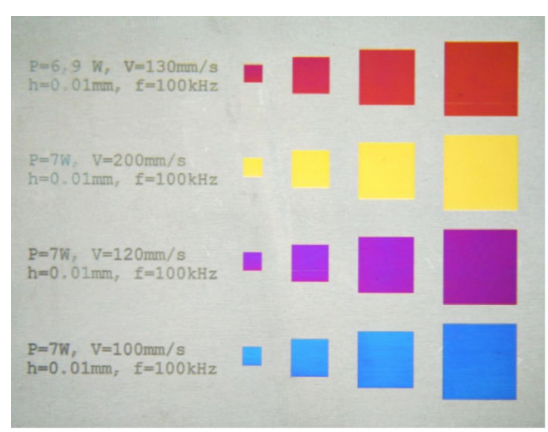

$\mathbf{a}$
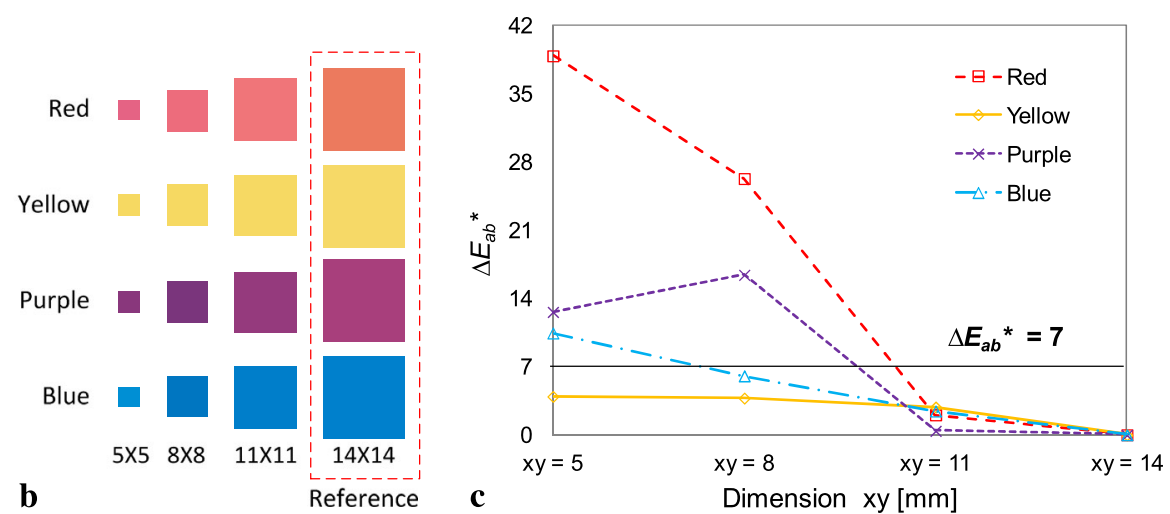

Fig. 10 The influence of size of marked field on the obtained colors (the reference parameters for all tested colors are given in Table 2, no. 1, 3-5): (a) photo of the test sample, (b) the color palette created based on the colorimetric measurements, (c) diagram of the $\Delta E_{a b}^{*}$ parameter

unit). Consequently, the stabilization of laser power at a level of a few times $10^{-3}$ or higher $(\ll 1 \%)$ is necessary in order to obtain the required color repeatability. Additionally, the operator of the system should be able to operate on "real values" of the optical power instead of "expected values." For each of the tested colors, the tolerances of both parameters (power and speed) were comparable.

In most laser marking systems with galvanometric scanning of the material, standard F-Theta lenses (not telecentric) are used. They enable focusing of the laser beam on a flat surface in a specified area, but do not provide a fixed geometry of the laser spot. As the laser beam is deflected off the axis, the beam angle moves away from normal. For such a case, the spot shape off the axis will be elongated (in the direction of beam deflection) proportionally to the output scan angle $\phi$. In addition, as a result of amendments in the angle of incidence of the laser beam on the material, the value of absorption will also be changed. Hence, it was necessary to determine the color changes as a function of the position of the marked field within the working area. The measurements taken for the red color are shown in Fig. 9. The results for other colors were comparable.

This value of color difference $\Delta E_{a b}^{*}$ is significantly smaller than in the cases considered previously. However, it can be further reduced by using a telecentric F-theta lens, where the output scan angle is always zero, or by simultaneous correction of laser power (proportional to the deflection angle of the beam). The very small asymmetry in $\Delta E_{a b}^{*}$ visible on the graph (Fig. 9c) may be caused by the nonideal laser beam spot shape $\left(M^{2}=1.5\right)$ and its eccentricity relative to the optical path of the BDU.

The last considered factor that might affect color reproducibility (through accumulative thermal effects) was the size of marked fields. The results are shown in Fig. 10. Similar trends (not value) in the $\Delta E_{a b}^{*}$ parameter are visible for all the tested colors. In the case of yellow, the fluctuation of $\Delta E_{a b}^{*}$ parameter that were noticed for up to eight times different sizes of the marked fields was below 3.9. The situation recorded for red and purple was different. A smaller marked field size shortens the time between two subsequent scanning lines. This, in turn, increases the sample temper- 


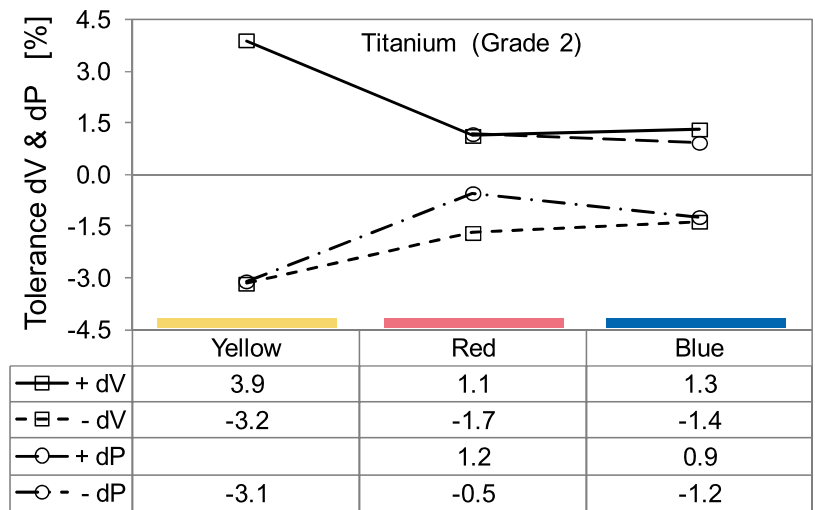

a

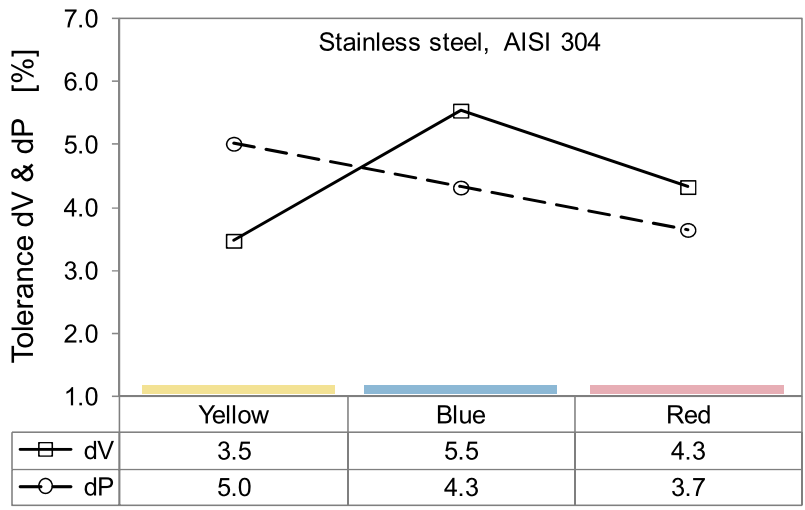

c

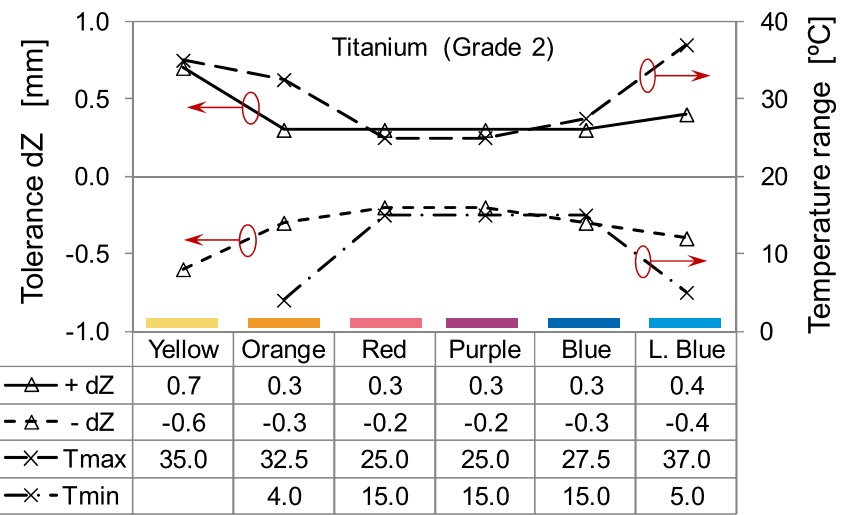

b

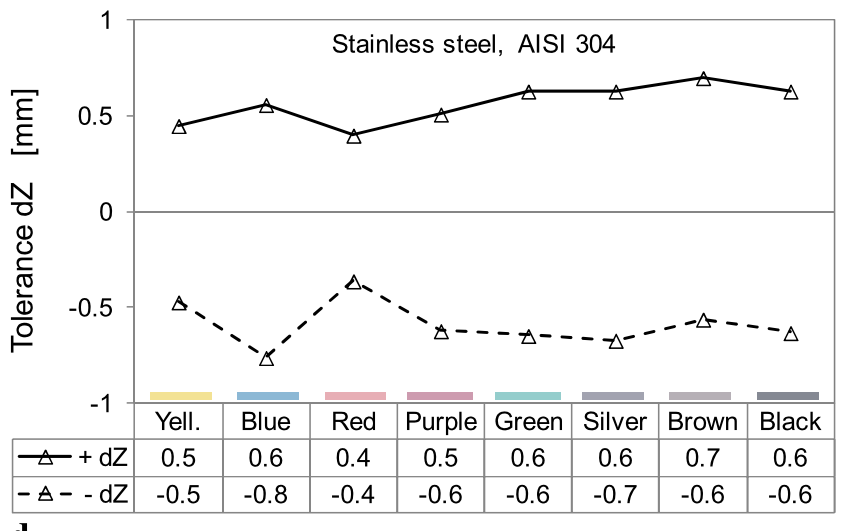

d

Fig. 11 Summary of selected results for process parameters tolerance enabling laser color marking with color difference $\Delta E_{a b}^{*} \leq 7:(\mathbf{a})$ and (b) for titanium, (c) and (d) for stainless steel AISI 304, based on previous research [18]

ature due to the thermal accumulative effect. Similarly to the previously studied case of the influence of temperature changes on the resulting sample color (Fig. 4), an analogous effect was obtained also here.

The impact of each of the process parameters was analyzed separately. For all those parameters which affect the color (taking into account the worst case), stability at a level of a few times $10^{-3}$ or higher is required. In reality, the color change effect is a superposition of all the possible (analyzed) factors. Overall, in order to achieve, in industrial conditions, stability of process parameters at such a required level, an active feedback loop is necessary. This can be done for example by measuring the actual laser output power, the temperature of marked sample, and the distance from the lens to the object. This is important because the required tolerance of sample position $(<0.2 \mathrm{~mm})$ is smaller than the accuracy of dimensions of many of the items to be marked. A change in this distance can also be compensated with a dynamic focusing unit. In the case of small deviations of the sample from the focal plane, the change in the laser fluence at the sample surface can be compensated (based on the information from the distance sensor) by correcting the optical power.

\section{Discussion of the results}

The results show that the tolerance of process parameters, enabling repeatable colors to be obtained highly depends on the specific color. Similar trends were observed in the study of stainless steel [18]. Selected results for both materials are shown in Fig. 11.

When comparing the results for both materials, two main differences are visible. The tolerances of optical power $d P$, scanning speed of the material $d V$ and position relative to the focal plane $d Z$ are on average 2-3 times lower for titanium than those for stainless steel. Moreover, and in contrast to titanium, the influence of sample temperature and dimensions of marked field on the obtained colors was negligible for stainless steel [18].

In order to clarify why the tolerance of process parameters depends on the specific color, an additional analysis was performed. Under a simplifying assumption that the titanium oxide film is a single homogeneous layer, reflectance characteristics for the oxide thickness varying from zero to $450 \mathrm{~nm}$ in steps of $2 \mathrm{~nm}$ were calculated. The complex refractive index values available in [29] for titanium, and in [30] for titanium dioxide were used in calculations. Next, the reflectance characteristics (calculated for the range of 

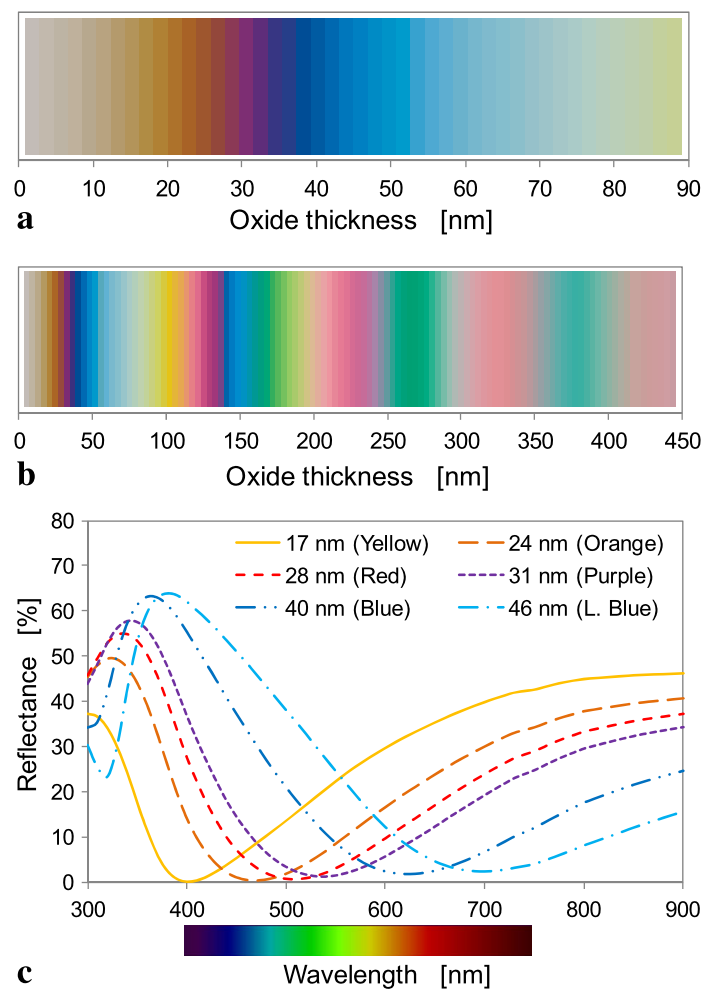

Fig. 12 The analysis of oxide film thickness: (a) and (b) illustrate the sequences of colors obtained as a function of oxide layer thickness in the range of $0-90 \mathrm{~nm}$ and $0-450 \mathrm{~nm}$, respectively, (c) reflectance versus wavelength for each of the analyzed colors

360-830 nm) for each oxide layer thickness were converted to the CIE $L^{*} a^{*} b^{*}$ color space. Colorimetric data enabled the presentation of their corresponding colors. The resulting colors arranged according to the oxide layer thickness are shown in Fig. 12a (for the range of 0-90 nm thick), and in Fig. 12b for the full range under analysis $(0-450 \mathrm{~nm})$. It is worth noting that the sequence of colors achieved as a function of the layer thickness (Fig. 12a) is in agreement with that obtained by increasing the laser fluence (Fig. 2c). By comparing the experimental data with calculations, the approximate oxide layer thicknesses for each color were determined. This analysis showed that the thickness of oxide formed by laser irradiation of titanium was in the range of 0-60 nm (the first possible sequences). In order to obtain the green color, for example, a layer about three times thicker is needed. The data shown in Fig. 12a and b are also in line with examples available in published literature [14, 31].

The characteristics of reflectance versus wavelength for each analyzed color and the corresponding oxide layer thicknesses are shown in Fig. 12c.

The resulting colorimetric data $\left(L^{*}, a^{*}\right.$, and $\left.b^{*}\right)$ for each oxide thickness (in increments of $2 \mathrm{~nm}$ ) allowed the calcu-
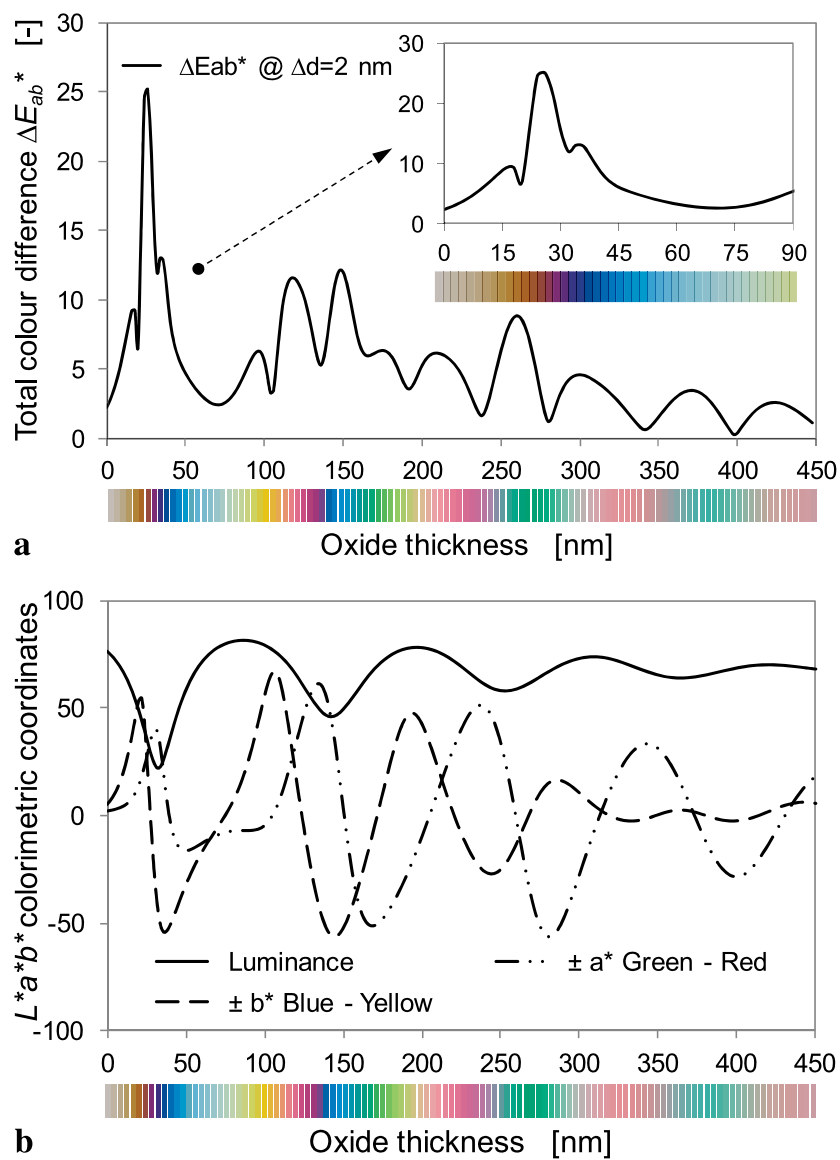

Fig. 13 The $C I E L^{*} a^{*} b^{*}$ color space representation: (a) total color difference $\Delta E_{a b}^{*}$ parameter calculated for the color changes caused by a $2 \mathrm{~nm}$ increase of the oxide thickness, (b) $C I E L^{*} a^{*} b^{*}$ colorimetric coordinates

lation of the total color difference $\Delta E_{a b}^{*}$ (Fig. 13a). Even though the increase of layer thickness was constant $(\Delta d=$ $2 \mathrm{~nm}$ ), the value of the color difference $\Delta E_{a b}^{*}$ was strongly dependent on the specific color, as shown in Fig. 13a.

As can be seen (Fig. 13a-enlarged area), the changes in $\Delta E_{a b}^{*}$ for the dark orange, red, or purple colors $(d \approx 25$ $30 \mathrm{~nm})$ are much greater than for the yellow $(d \approx 17 \mathrm{~nm})$ or the light blue $(d \approx 46 \mathrm{~nm})$ color, which was confirmed by the measurements. Of course, the $\Delta E_{a b}^{*}$ parameter value should be considered as relative (they depend on the assumed thickness). The rapid increase in the color difference results from the significant changes in the colorimetric coordinates (Fig. 13b).

What is also apparent is that, with the increase of oxide thickness, the colors become "less monochromatic." Above the thickness of $250 \mathrm{~nm}$, it is only possible to obtain colors similar to pink or turquoise. Finally, the amplitude changes of colorimetric coordinates decrease with increasing oxide thickness. 


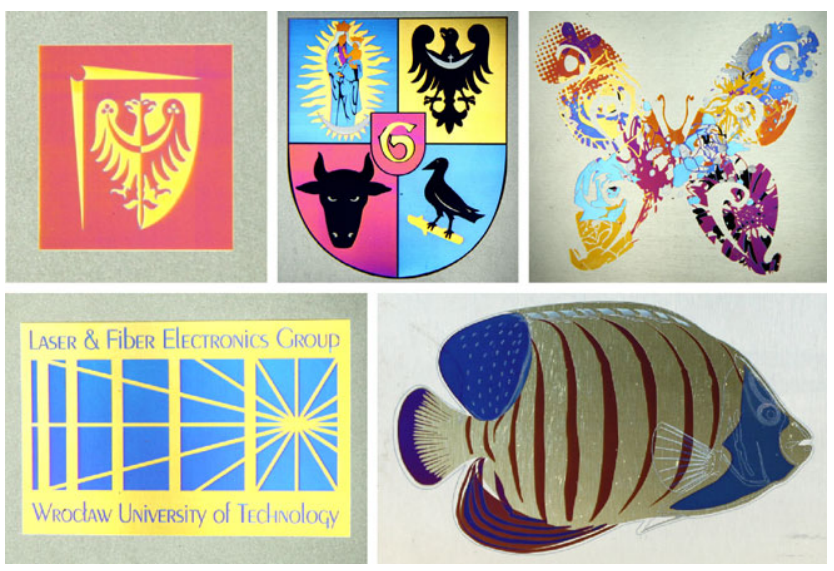

Fig. 14 Examples of color laser marking on titanium. Trademarks and trade names are the exclusive property of their respective owners. They are used only to demonstrate the possibilities of laser color marking

\section{Examples of color laser marking}

Laser color marking of metals can be important in advertising (having the logo appear in a company's brand color), electronics, automotive industry, jewelry [7, 8], art (new designs), and in many other fields. Some examples of laserinduced color marking on titanium are shown in Fig. 14.

\section{Conclusion}

This paper presents the results of the measurement and analyses of how the laser marking process parameters influence the colors created on titanium. The investigations were performed using a commercially available industrial pulsed fiber laser. For objective assessment of color changes, a CIE color difference formula $\Delta E_{a b}^{*}$ was used. Colors were considered to be similar if the value of the color difference parameter $\Delta E_{a b}^{*}$ did not exceed 7 .

We found that all the analyzed process parameters (i.e., initial temperature of the material, laser fluence, size of the marked area and position of the marked surface, relative to both the focal plane and the center of the working field of the system) affect the reproducibility of the color obtained. Obviously, pulse duration, pulse repetition rate and line spacing (hatching) also significantly change the resulting color, but these parameters were kept constant. In practice, the stability of these values poses no problem.

The reproducibility of laser color marking of titanium, with the assumed value of color difference $\Delta E_{a b}^{*}$ requires (taking into account the worst case) the maintenance of the laser fluence fluctuation $<1 \%$, the initial temperature of the material with accuracy $< \pm 5{ }^{\circ} \mathrm{C}$, and the position of the sample relative to the focal plane $< \pm 0.2 \mathrm{~mm}$ independently.
We found that the tolerance of process parameters is highly dependent on the particular color. Colorimetric analysis showed that this results from the nature of changes of the reflectance spectra as a function of layer thickness (at the specific layer thickness).

We also compared the results obtained for titanium with those for stainless steel from previous research. The tolerances of all the process parameters were on average 2-3 times lower than those for stainless steel. Moreover, and in contrast to titanium, the influence of sample temperature and of dimensions of the marked field on the colors obtained was negligible for stainless steel [18].

In order to achieve, in industrial conditions, the stability of the process parameters at the required level $\left(\Delta E_{a b}^{*} \leq 7\right)$, it is necessary to use an active feedback loop.

Acknowledgements This research was supported by Wroclaw Research Centre EIT + under the project "The Application of Nanotechnology in Advanced Materials"-NanoMat (POIG.01.01.02-02002/08) financed by the European Regional Development Fund (Innovative Economy Operational Programme, 1.1.2).

The authors would like to thank Łukasz Łazarek, M.Sc. for his assistance with the preparation of some of the test samples.

Open Access This article is distributed under the terms of the Creative Commons Attribution License which permits any use, distribution, and reproduction in any medium, provided the original author(s) and the source are credited.

\section{References}

1. Ch.J. Barbe, F. Arendse, P. Comte, M. Jirousek, F. Lenzmann, V. Shklover, M. Gratzel, Nanocrystalline titanium oxide electrodes for photovoltaic applications. J. Am. Ceram. Soc. 80, 3157-3171 (1997)

2. A. Fujishima, T.N. Rao, D.A. Tryk, Titanium dioxide photocatalysis. J. Photochem. Photobiol., C, Photochem. Rev. 1, 1-21 (2000)

3. G. Eranna, B.C. Joshi, D.P. Runthala, R.P. Gupta, Oxide materials for development of integrated gas sensors-a comprehensive review. Crit. Rev. Solid State Mater. Sci. 29, 111-188 (2004)

4. Y.T. Sula, The significance of the surface properties of oxidized titanium to the bone response: special emphasis on potential biochemical bonding of oxidized titanium implant. Biomaterials 24 (2003)

5. M.H. Suhail, G.M. Rao, S. Mohan, Dc reactive magnetron sputtering of titanium-structural and optical characterization of TQ films. J. Appl. Phys. 71, 1421 (1992)

6. A. Pérez del Pino, P. Serra, J.L. Morenza, Oxidation of titanium through Nd_YAG laser irradiation. Appl. Surf. Sci. 197-198, 887890 (2002)

7. M.V. Diamanti, B. Del Curto, M.P. Pedeferri, Interference colors of thin oxide layers on titanium. Color Res. Appl. 33, 221-228 (2008)

8. S. O'Hana, A.J. Pinkerton, K. Shoba, A.W. Gale, L. Li, Laser surface colouring of titanium for contemporary jewelery. Surf. Eng. 24, 147-153 (2008)

9. H. Takikawa, T. Matsui, T. Sakakibara, A. Bendavid, P.J. Martin, Properties of titanium oxide film prepared by reactive cathodic vacuum. Thin Solid Films 348, 145-151 (1999) 
10. K.S. Teng, J.-L. Delplancke, J. Zhang, T.J. O’keefe, Electrochemical characterization of copper deposited on plasma and thermally modified titanium surfaces. Metall. Mater. Trans. B 29B (1998)

11. C. Garapon, C. Champeaux, J. Mugnier, G. Panczer, P. Marchet, A. Catherinot, B. Jacquier, Preparation of TiO, thin films by pulsed laser deposition for waveguiding applications. Appl. Surf. Sci. 96-98, 836-841 (1996)

12. J.H. Lee, G.E. Jang, Y.H. Jun, Investigation and evaluation of structural color of $\mathrm{TiO}_{2}$ coating on stainless steel. Ceram. Int. 38 (2012)

13. H. Dong, T. Bell, Enhanced wear resistance of titanium surfaces by a new thermal oxidation treatment. Wear 238, 131-137 (2000)

14. Á. Perez del Pino, J.M. Fernandez-Pradas, P. Serra, J.L. Morenza, Coloring of titanium through laser oxidation: comparative study with anodizing. Surf. Coat. Technol. 187, 106-112 (2004)

15. L. Lavisse, D. Grevey, C. Langlade, B. Vannes, The early stage of the laser-induced oxidation of titanium substrates. Appl. Surf. Sci. 186, 150-155 (2002)

16. L. Baufay, F.A. Houle, R.J. Wilson, Optical self-regulation during laser-induced oxidation of copper. J. Appl. Phys. 61, 4640 (1987)

17. Z.L. Li, H.Y. Zheng, K.M. Teh, Y.C. Liu, G.C. Lim, H.L. Seng, N.L. Yakovlev, Analysis of oxide formation induced by UV laser coloration of stainless steel. Appl. Surf. Sci. 256, 1731-1734 (2009)

18. A.J. Antończak, D. Kocoń, M. Nowak, P. Kozioł, K.M. Abramski, Laser-induced colour marking-Sensitivity scaling for a stainless steel. Appl. Surf. Sci. 264 (2013)

19. S. Fujimoto, K. Tsujino, T. Shibata, Growth and properties of Cr-rich thick and porous oxide films on Type 304 stainless steel formed by square wave potential pulse polarisation. Electrochim. Acta 47, 543-551 (2001)

20. A.Y. Vorobyev, C. Guo, Colorizing metals with femtosecond laser pulses. Appl. Phys. Lett. 92, 041914 (2008)

21. B. Dusser, Z. Sagan, H. Soder, N. Faure, J.P. Colombier, M. Jourlin, E. Audouard, Controlled nanostructrures formation by ul- tra fast laser pulses for color marking. Opt. Express 18, 2913-2924 (2010)

22. A.A. Ionin, S.I. Kudryashov, S.V. Makarov, L.V. Seleznev, D.V. Sinitsyn, E.V. Golosov, O.A. Golosova, Y.R. Kolobov, A.E. Ligachev, Femtosecond laser color marking of metal and semiconductor surfaces. Appl. Phys. A 107 (2012)

23. E.B. Barmina, E. Stratakis, C. Fotakis, G.A. Shafeev, Generation of nanostructures on metals by laser ablation in liquids: new results. Quantum Electron. 40, 1012 (2010)

24. K. McLaren, XIII-The development of the CIE $1976\left(L^{*} a^{*} b^{*}\right)$ uniform colour space and colour-difference formula. J. Soc. Dyers Colour. 92, 338-341 (1976)

25. F. Bunting, The Color Shop, Color Primer: An Introduction to the History of Color, Color Theory, and Color Measurement (Light Source Computer Images, Inc., An X-Rite Company, Grandville, 1998), p. 44

26. D.P. Adams, V.C. Hodges, D. Hirschfeld, M.A. Rodriguez, J.P. McDonald, P.G. Kotula, Nanosecond pulsed laser irradiation of stainless steel 304l: oxide growth and effects on underlying metal. Surf. Coat. Technol. (2013, in press)

27. Y.S. Tian, C.Z. Chen, S.T. Li, Q.H. Huo, Research progress on laser surface modification of titanium alloys. Appl. Surf. Sci. 242, 177-184 (2005)

28. http://www.ipgphotonics.com/Collateral/Documents/English-US/ YLP_Series.pdf (datasheets of the YLP Series fiber laser)

29. D.W. Lynch, C.G. Olson, J.H. Weaver, Optical properties of Ti, Zr, and $\mathrm{Hf}$ from 0.15 to $30 \mathrm{eV}$. Phys. Rev. B 11, 3617-3624 (1975)

30. J.R. Devore, Refractive indices of rutile and sphalerite. J. Opt. Soc. Am. B, Opt. Phys. 41, 416-417 (1951)

31. M.V. Diamanti, B. Del Curto, V. Masconale, M. Pedeferri, Production and anodic colouring of newly-designed titanium jewels. Colour Des. Creat. 5, 1-9 (2010) 